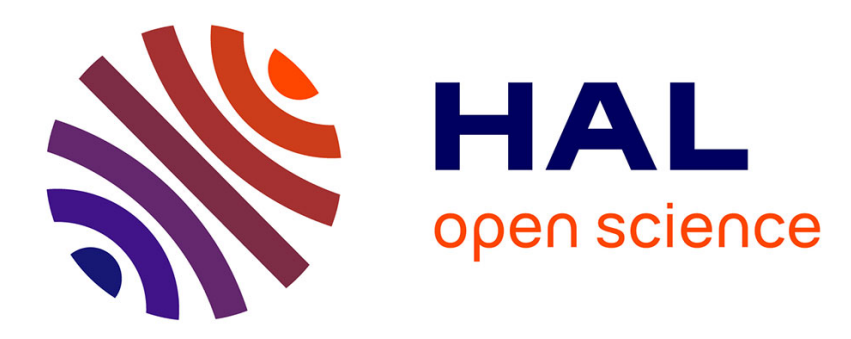

\title{
Nonlinear categorization of the energetic-beam-driven instability with drag and diffusion
}

\author{
Maxime Lesur, Y. Idomura
}

\section{To cite this version:}

Maxime Lesur, Y. Idomura. Nonlinear categorization of the energetic-beam-driven instability with drag and diffusion. Nuclear Fusion, 2012, 52 (9), pp.094004. 10.1088/0029-5515/52/9/094004 . hal01969729

\section{HAL Id: hal-01969729 \\ https://hal.science/hal-01969729}

Submitted on 14 Jan 2019

HAL is a multi-disciplinary open access archive for the deposit and dissemination of scientific research documents, whether they are published or not. The documents may come from teaching and research institutions in France or abroad, or from public or private research centers.
L'archive ouverte pluridisciplinaire HAL, est destinée au dépôt et à la diffusion de documents scientifiques de niveau recherche, publiés ou non, émanant des établissements d'enseignement et de recherche français ou étrangers, des laboratoires publics ou privés. 
Nonlinear categorization of the energetic-beam-driven instability with drag and diffusion

This article has been downloaded from IOPscience. Please scroll down to see the full text article.

2012 Nucl. Fusion 52094004

(http://iopscience.iop.org/0029-5515/52/9/094004)

View the table of contents for this issue, or go to the journal homepage for more

Download details:

IP Address: 133.5.178.253

The article was downloaded on 04/09/2012 at 01:53

Please note that terms and conditions apply. 


\title{
Nonlinear categorization of the energetic-beam-driven instability with drag and diffusion
}

\author{
M. Lesur ${ }^{1, a}$ and Y. Idomura ${ }^{2}$ \\ ${ }^{1}$ National Fusion Research Institute, Gwahangno 113, Yusung-gu, Daejeon 305-333, Korea \\ 2 Japan Atomic Energy Agency, Obuchi-Omotedate 2-166, Rokkasho, Kamikita, Aomori \\ 039-3212, Japan \\ E-mail: maxime.lesur@polytechnique.org
}

Received 18 December 2011, accepted for publication 3 July 2012

Published 3 September 2012

Online at stacks.iop.org/NF/52/094004

\begin{abstract}
The Berk-Breizman (BB) extension of the bump-on-tail instability includes a finite, fixed wave damping $\left(\gamma_{\mathrm{d}}\right)$, and a collision operator with drag $\left(v_{\mathrm{f}}\right)$ and diffusion $\left(v_{\mathrm{d}}\right)$. The BB model is applied to a one-dimensional plasma, to investigate the kinetic nonlinearities, which arise from the resonance of a single electrostatic wave with an energeticparticle beam. For a fixed value of the linear drive normalized to the linear frequency, $\gamma_{\mathrm{L} 0} / \omega_{0}=0.1$, the long-time nonlinear evolution is systematically categorized as damped, steady-state, periodic, chaotic and chirping. The chirping regime is sub-categorized as periodic, chaotic, bursty and intermittent. Up-down asymmetry and hooked chirping branches are also categorized. For large drag, holes with quasi-constant velocity are observed, in which case the solution is categorized into steady, wavering and oscillating holes. Two complementary parameter spaces are considered: (1) the $\left(\gamma_{\mathrm{d}}, \nu_{\mathrm{d}}\right)$ space for fixed $\nu_{\mathrm{d}} / \nu_{\mathrm{f}}$ ratios; (2) the $\left(\nu_{\mathrm{f}}, \nu_{\mathrm{d}}\right)$ space for fixed $\gamma_{\mathrm{d}} / \gamma_{\mathrm{L} 0}$ ratios, close to and far from marginal stability. The presence of drag and diffusion (instead of a Krook model) qualitatively modifies the nonlinear bifurcations. The bifurcations between steady-state, periodic and steady-hole solutions agree with analytic theory. Moreover, the boundary between steady and periodic solutions agrees with analytic theory. Nonlinear instabilities are found in both subcritical and barely unstable regimes. Quasi-periodic chirping is shown to be a special case of bursty chirping, limited to a region relatively far from marginal stability.
\end{abstract}

(Some figures may appear in colour only in the online journal)

\section{Introduction}

A major concern in burning plasmas is that high-energy ions can excite plasma instabilities in the frequency range of Alfvén eigenmodes (AEs), which significantly enhance their transport. Transport and loss of fast particles depend on both the nonlinear saturation amplitude and the kind of nonlinear behaviour. Many qualitatively different nonlinear regimes have been observed in experiments [1], including a zoo of spectral components whose frequency shifts on a time scale much smaller than the profiles' evolution time scale (nonlinear chirping). The behaviours of these spectral components are qualitatively diverse in terms of their intermittency [2-4], their monotonicity in frequency shift $[5,6]$, their asymmetry [5, 7], and whether frequency shifting branches end as a continuous mode [8] or not. This work aims at defining and categorizing each nonlinear regime in a systematic manner. Such a

a Present address: Itoh Research Center for Plasma Turbulence, Kasuga, Kasuga Koen 6-1, 816-8580, Kyushu University, Japan. categorization indicates how experimental input parameters may be adjusted to change the nonlinear behaviour. For instance, since Alfvén avalanches are often associated with chirping bursts, rather than continuous modes, a good strategy would be to avoid chirping bursts.

Near the resonant surface, it is possible to obtain a new set of variables in which the three-dimensional (3D) plasma is described by a one-dimensional Hamiltonian in two conjugated variables [9-11], if we assume an isolated single resonance. In this sense, the problem of AEs is homothetic to the well-known paradigm of a single mode bump-on-tail instability. The BerkBreizman (BB) problem $[9,10,12,13]$ is a generalization of the bump-on-tail problem, where we take into account an external wave damping accounting for background dissipative mechanisms at a rate $\gamma_{\mathrm{d}}$, and a collision operator. Observed quantitative similarities between the BB nonlinear theory and both global simulations $[11,14]$ and experiments $[15,16]$ are an indication of the validity of the aforementioned reduction of dimensionality. 
The BB model has been extensively studied over the last few decades, in the case where collisions are modelled by a simple Krook-like operator with a collision frequency $v_{\mathrm{a}}$, or by a velocity-diffusion operator. It has been predicted that three kinds of behaviours emerge, namely steady-state, periodic or chaotic responses, depending on the strength of each factor $[9,10,13]$. In addition, chaotic solutions can display shifting of the mode frequency (chirping). In general, two branches coexist, with their frequency sweeping downwardly (downchirping) for one, upwardly (up-chirping) for the other, as pairs of holes and clumps in the distribution evolve [17-19]. A systematic categorization of these nonlinear regimes in the $\left(\gamma_{\mathrm{d}}, v_{\mathrm{a}}\right)$ parameter space for a fixed initial velocity distribution has been performed numerically [20,21]. However, recently, Lilley and Lesur have shown that the inclusions in the collision operator of dynamical friction, or drag, and diffusion have a strong impact on the nonlinear behaviour, and is necessary to qualitatively reproduce experimental chirping AEs [22,23].

In this work, we name and define new sub-categories of chirping, namely periodic, bursty, intermittent, chaotic, steady hole, wavering hole, oscillating hole, hooked and subcategories that characterize the asymmetry between downward and upward frequency sweeping. We develop an algorithm to systematically determine the category to which a solution belongs. This algorithm is applied to long-time Vlasov simulations of the BB model with drag and diffusion. If needed, it could be adapted to analyse experimental data. Our simulation parameters correspond to a cold bulk, weak warm beam plasma, with a constant slope around the resonant velocity and a fixed value of the linear drive normalized to the linear wave frequency, $\gamma_{\mathrm{L} 0} / \omega_{0}=0.1$, which is experimentally relevant $[6,24,25]$. We study two complementary parameter spaces: (1) the $\left(\gamma_{\mathrm{d}}, v_{\mathrm{d}}\right)$ space for fixed drag/diffusion ratios, $v_{\mathrm{d}} / \nu_{\mathrm{f}}=1-5$; (2) the $\left(v_{\mathrm{f}}, v_{\mathrm{d}}\right)$ space for fixed damping rates, close to and relatively far from marginal stability, $\gamma / \gamma_{\text {L } 0}=0.05-0.4$.

When $v_{\mathrm{f}} \ll v_{\mathrm{d}}$, the phase diagram is qualitatively similar to what was obtained with Krook collisions, although chirping solutions can be intermittent, bursty or periodic, in addition to the chaotic behaviour found in the Krook case. We show that quasi-periodic chirping is a special case of bursty chirping, limited to a region where $\gamma_{\mathrm{d}} / \gamma_{\mathrm{L} 0}=0.2$ 0.7. The presence of significant drag qualitatively modifies the nonlinear bifurcations. Steady-state, periodic and chaotic solutions, which are devoid of significant phase-space structure dynamics, are replaced by long-lived phase-space holes. The periodic chirping regime almost disappears. We confirm that steady-state solutions only exist for collision frequencies above a threshold predicted by analytic theory [22]. Moreover, the boundary between steady and periodic solutions agrees with analytic theory when the system is close to marginal stability. Nonlinear instabilities are found in both subcritical $(\gamma<0)$ and barely unstable $\left(\gamma \ll \gamma_{\mathrm{L} 0}\right)$ regimes. We did not find any solution for which downward chirping is dominant.

\section{The BB model}

\subsection{Model description}

We assume a single electrostatic wave, with a wave number $k$. The assumption that the electric field is sinusoidal corresponds to the situation of a single resonance, which is selected by the geometry in more complex systems. It is appropriate to normalize time to the plasma frequency $\omega_{\mathrm{p}}$, distance to the wave number $k$, density to the total plasma density $n_{0}$ and electric field to $m \omega_{\mathrm{p}}^{2} /(e k)$, where $e$ and $m$ are the particle charge and mass, and $\omega_{\mathrm{p}}^{2} \equiv n_{0} e^{2} /\left(\epsilon_{0} m\right)$. We adopt a perturbative approach, and cast the BB model in a reduced form, which describes the time evolution of the beam particles only [26]. The main hypothesis in this approach is that the bulk particles interact adiabatically with the wave, so that their contribution to the Lagrangian can be expressed as a part of the electric field. In this model, the linear frequency of the wave is imposed as $\omega_{0}=\omega_{\mathrm{p}}$, which implies that in practice, time is normalized to the linear frequency of the wave (without nonlinear modification). Even when chirping occurs, $\omega_{0}$ does not change. Chirping (existence of a significant spectral component at finite $\left.\delta \omega \equiv \omega-\omega_{0}\right)$ is due to the nonlinear evolution of the amplitude and phase of the wave. The evolution of the beam distribution, $f(x, v, t)$, is given by a kinetic equation,

$$
\frac{\partial f}{\partial t}+v \frac{\partial f}{\partial x}+\tilde{E} \frac{\partial f}{\partial v}=v_{\mathrm{f}}^{2} \frac{\partial\left(f-f_{0}\right)}{\partial v}+v_{\mathrm{d}}^{3} \frac{\partial^{2}\left(f-f_{0}\right)}{\partial v^{2}}
$$

where the rhs is a collision operator, which includes both drag and diffusion, $f_{0}(v)$ is the initial velocity distribution and the pseudo-electric field $\tilde{E}$ is defined as

$$
\tilde{E}(x, t) \equiv Q(t) \cos (x-t)-P(t) \sin (x-t) .
$$

The evolution of the pseudo-electric field is given by

$$
\begin{aligned}
& \frac{\mathrm{d} Q}{\mathrm{~d} t}=-\frac{1}{2 \pi} \int f(x, v, t) \cos (x-t) \mathrm{d} x \mathrm{~d} v-\gamma_{\mathrm{d}} Q, \\
& \frac{\mathrm{d} P}{\mathrm{~d} t}=+\frac{1}{2 \pi} \int f(x, v, t) \sin (x-t) \mathrm{d} x \mathrm{~d} v-\gamma_{\mathrm{d}} P .
\end{aligned}
$$

The term proportional to $\gamma_{\mathrm{d}}$ is an external wave damping, which is a model for all linear dissipative mechanisms of the wave energy to the background plasma [26]. Equations (3) and (4) are designed so that $\mathrm{d} W / \mathrm{d} t=P_{\mathrm{h}}-2 \gamma_{\mathrm{d}} W$, where $W=$ $\left(Q^{2}+P^{2}\right) / 2$ is the total wave energy, including the sloshing energy, and $P_{\mathrm{h}}$ is the power the energetic particles transfer to the wave.

We refer to the above reduced model as the $\delta f \mathrm{BB}$ model. Compared with the full- $f$ model, the $\delta f$ model does not take into account effects of the time evolution of the bulk particle velocity distribution. In addition, the $\delta f$ model assumes a constant total number of energetic particles.

\subsection{Numerical simulations}

In [21], we described the kinetic code COBBLES (COnservative Berk-Breizman semi-Lagrangian Extended Solver), capable of long-time simulations of the $\delta f$ BB model with a Krook collision operator. COBBLES was extended to include drag and diffusion in the collision operator. We verify the latter implementation in appendix A by comparing simulations with a perturbative theory for the nonlinear saturation of solutions to the BB model with drag and diffusion, which was developed by Lilley [22]. This also constitutes 


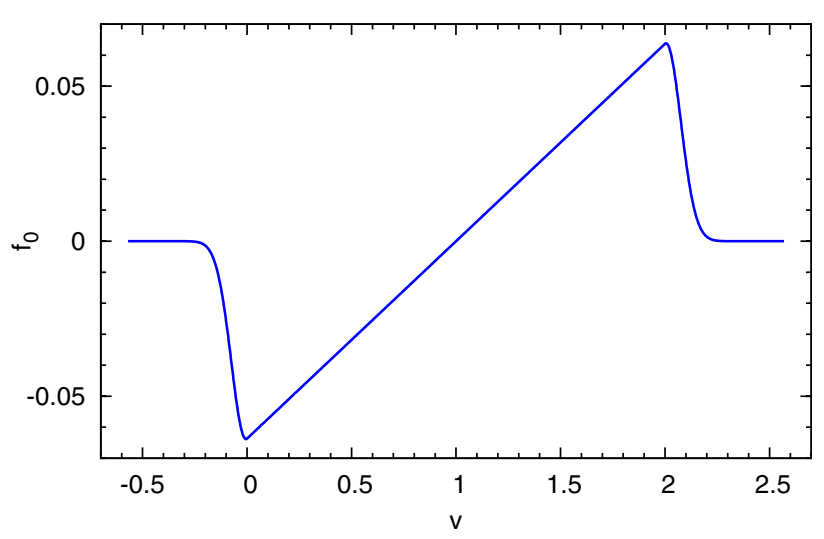

Figure 1. Assumed initial velocity distribution, with $\gamma_{\mathrm{L} 0}=0.1$.

the first numerical validation of the above theory by direct simulations of the non-truncated BB equations.

Hereafter, the initial velocity distribution $f_{0}$ is designed with a constant slope in the region where chirping occurs (as long as $\delta \omega / \omega_{0}<1$, which is consistent with experiments). We define $\gamma_{\mathrm{L} 0}$ as a measure of the slope of $f_{0}$ at the resonant velocity $v=1$ :

$$
\left.\gamma_{\mathrm{L} 0} \equiv \frac{\pi}{2} \frac{\partial f_{0}}{\partial v}\right|_{v=1} .
$$

In the collisionless limit, when $\gamma \ll \omega_{0}$, the linear growth rate reduces to $\gamma=\gamma_{\mathrm{L} 0}-\gamma_{\mathrm{d}}$.

We arbitrarily choose

$$
\frac{\pi}{2 \gamma_{\mathrm{L} 0}} f_{0}(v)=\left\{\begin{array}{l}
-\frac{v 2-v 1}{2} \exp \left[\sigma^{2}-\left(\frac{v-v 1}{v_{\sigma}}+\sigma\right)^{2}\right] \\
\quad \text { if } v<0, \\
v-1 \\
\text { if } 0 \leqslant v \leqslant 2, \\
+\frac{v 2-v 1}{2} \exp \left[\sigma^{2}-\left(\frac{v-v 2}{v_{\sigma}}+\sigma\right)^{2}\right] \\
\text { if } v>2,
\end{array}\right.
$$

with $\gamma_{\mathrm{L} 0}=0.1, v_{1}=0, v_{2}=2, \sigma=0.05$ and $v_{\sigma}=$ $\sigma\left(v_{2}-v_{1}\right)$. The linear drive $\gamma_{\mathrm{L}}$, which is defined as the linear growth rate in the absence of damping and collision, is $\gamma_{\mathrm{L}} / \omega_{0}=0.09$. This is consistent with energetic-particledriven experiments, where $\gamma_{\mathrm{L}} / \omega_{0}$ is estimated within the range $\sim 0.1-30 \%$. The function $f_{0}$ is shown within the simulation domain $-0.57<v<2.57$ in figure 1 . It is acknowledged that deviations from a constant-slope distribution are expected to affect the nonlinear behaviour. Thus, when discussing experiments, our analysis must be understood as an ideal case where the energetic-particle distribution can be modelled with a constant slope in a neighbourhood of the resonant velocity, where the size of this neighbourhood depends on the domain spanned by resonant wave-particle interactions and evolving phase-space structures.

The electric field amplitude is measured by the bounce frequency $\omega_{\mathrm{b}}=\left(Q^{2}+P^{2}\right)^{1 / 4}$ of particles that are deeply trapped in the electrostatic potential. As the initial condition we apply a small perturbation, $f(x, v, t=0)=f_{0}(v)(1+$ $\epsilon \cos x)$, with $\epsilon=10^{-5}\left(\omega_{\mathrm{b}} / \gamma_{\mathrm{L} 0}=0.03\right)$, unless stated otherwise. The initial values of $Q$ and $P$ are given by solving Poisson's equation. All simulations are performed with at least
$N_{x}=128$ and $N_{v}=2048$ grid points in configuration space and velocity space, respectively, and with a time-step width $\Delta t=0.1$. If the electric field amplitude grows to large values, where numerical convergence conditions (such as the CourantFriedrichs-Lewy condition) are not satisfied, then the numbers of grid points are doubled, the time-step width is halved and the simulation starts again from $t=0$.

\section{Nonlinear behaviour bifurcations}

\subsection{Categorization}

We consider five main categories for the time evolution of the instability. The category is obtained by an analysis of $\omega_{\mathrm{b}}(t)$, and a spectrogram $\mathcal{P}(\delta \omega, t)$ of $\tilde{E}(0, t)$. Since we want to categorize the time-asymptotic behaviour, we restrict our analysis to a time interval that starts well after the end of the linear phase and the nonlinear saturation, ignoring the transient evolution. A numerical solution is defined as

1. Damped: if the asymptotic-time limit of $\omega_{\mathrm{b}}(t)$ is zero;

2. Chirping: if there is a spectral component in $\mathcal{P}$ with a significant amplitude whose frequency significantly deviates from the linear mode frequency;

3. Steady state: if the asymptotic-time limit of $\omega_{\mathrm{b}}(t)$ is finite;

4. Periodic: if for large enough $t$ there is a period $\tau$ for which $\omega_{\mathrm{b}}(t+\tau) \rightarrow \omega_{\mathrm{b}}(t)$

5. Chaotic: if $\omega_{\mathrm{b}}(t)$ is bounded, but does not satisfy one of the previous conditions.

The categories 1., 3., 4. and 5. were defined by Vann [20]. Each numerical solution is systematically categorized by an algorithm, which follows a decision tree based on the one developed by Vann. This algorithm was described and benchmarked in [21]. Here we use the same algorithm, with two slight improvements, which are described in appendix B. We also changed the order in the decision tree: the test for chirping is moved up to the second position. This test must be done early because chirping solutions can satisfy the criteria that define steady-state, periodic or chaotic behaviours.

We further categorize the repetitiveness, asymmetry and shape of chirping solutions into qualitatively distinguishable sub-categories. The following three sub-categorizations are obtained by an analysis of $\mathcal{P}$. Firstly, we define a chirping solution as

4.A. Long-lived hole (clump): if the spectrogram is almost never quiescent, and the average duration of a chirping branch is much larger than the time it takes to reach its maximum frequency shift. If long-lived chirping branches dominate the $\delta \omega>0(\delta \omega<0)$ part of $\mathcal{P}$, the category is long-lived hole (clump).

(a) Steady hole (clump): if the asymptotic-time limit of the frequency shift of the dominant branch is finite;

(b) Oscillatory hole (clump): if for large enough $t$ there is a period $\tau$ for which the frequency shift of the dominant branch, $\delta \omega(t)$, satisfies $\delta \omega(t+\tau) \rightarrow \delta \omega(t)$. Also called snaking;

(c) Wavering hole (clump): otherwise;

4.B. Periodic chirping: if for large enough $t$ there is a period $\tau$ for which the whole spectrogram, $\mathcal{P}(\delta \omega, t)$, satisfies $\mathcal{P}(\delta \omega, t+\tau) \rightarrow \mathcal{P}(\delta \omega, t)$ 
4.C. Bursty chirping: if the spectrogram consists mainly of alternating quiescent and non-quiescent phases, where each non-quiescent phase comprises one and no more than one major chirping branch;

4.D. Intermittent chirping: if the spectrogram mainly consists of alternating quiescent and non-quiescent phases, but each non-quiescent phase may comprise several major chirping branches;

4.E. Chaotic: if the spectrogram is almost never quiescent and without long-lived hole or clump, or if the spectrogram is a mix of the above categories.

Here, major chirping branch refers to a chirping branch that reaches a frequency shift close to the maximum reached by any chirping branch after the initial transient phase. Quiescent phases refer to time intervals where $\mathcal{P}(\delta \omega, t)$ is much smaller than the maximum of the whole spectrogram (after the transient phase). The difference between a steady-state solution and a steady-hole solution is that the latter satisfies the criterion for chirping. The same difference discriminate periodic from oscillatory hole, as well as chaotic from wavering hole. Although oscillatory hole solutions may satisfy the criteria for periodic chirping solutions, the latter do not present any longlived hole or clump.

Secondly, we categorize the asymmetry between upward and downward sweeping. We distinguish

- Upward chirping dominant: if the total power in the $\delta \omega>0$ part of the spectrogram is significantly larger than the total power in the $\delta \omega<0$ part;

- Upward chirping only: if the criterion for upward dominant is satisfied and the maximum frequency shift in the $\delta \omega<0$ direction is insignificant;

- Downward chirping dominant: if the total power in the $\delta \omega<0$ part of the spectrogram is significantly larger than the total power in the $\delta \omega>0$ part;

- Downward chirping only: if the criterion for downward dominant is satisfied and the maximum frequency shift in the $\delta \omega>0$ direction is insignificant;

- Symmetric: if none of the above conditions is satisfied.

Note that we choose to categorize asymmetry, not in terms of the amplitude of frequency shift, but rather in terms of the importance of phase-space structures. We do not categorize the asymmetry for long-lived holes or clumps, since holes belong to the $\delta \omega>0$ side, while clumps belong to the $\delta \omega<0$ side. We make an arbitrary distinction between upward (downward) chirping dominant and upward (downward) chirping only, because it may be useful for experiments, where each case can clearly be identified, and where phenomena that are not included in the BB model could be responsible for a physically meaningful distinction. Threshold parameters for each category is given in section B.3 in the appendix.

Thirdly, we categorize whether the end of chirping branches is hooked or not,

- Hooked: if most of the major chirping branches change their sweeping direction after having reached their maximum frequency shift;

- Not hooked: otherwise.
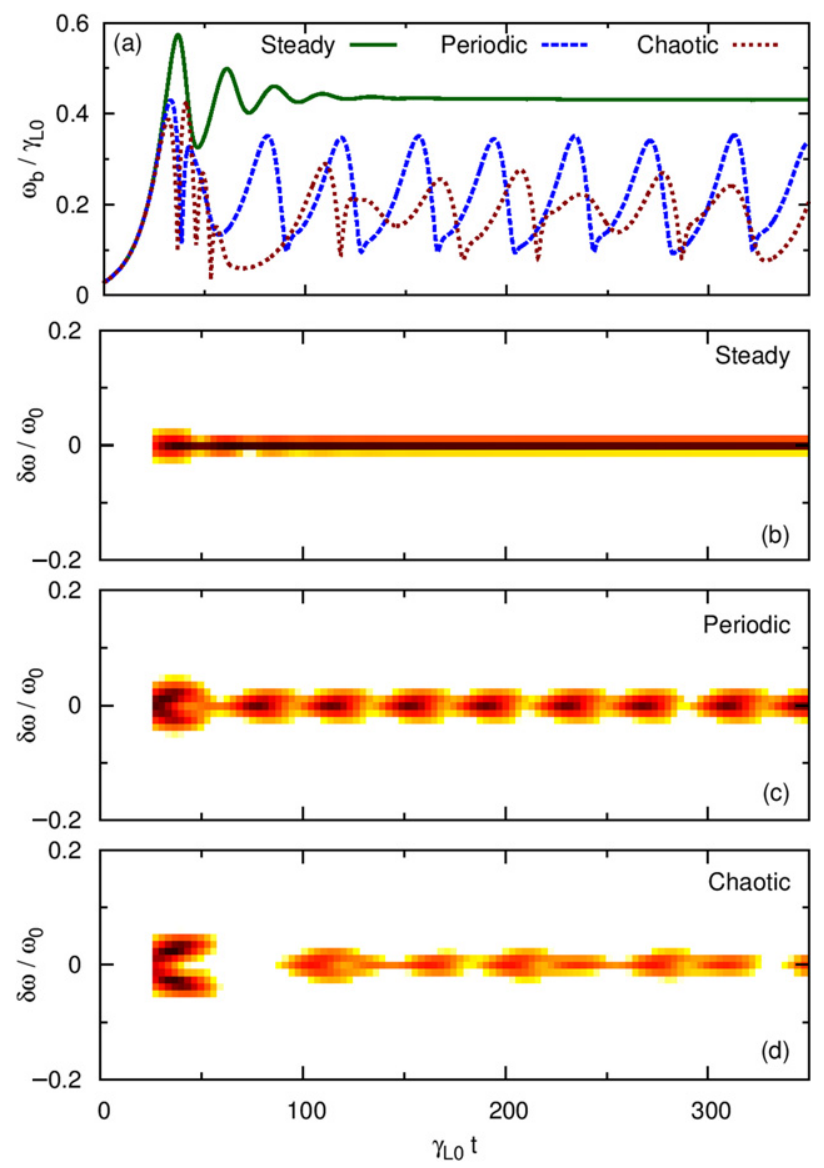

Figure 2. (a) Time series of electric field amplitude and $(b),(c),(d)$ spectrograms for typical non-chirping solutions with $\gamma_{\mathrm{d}} / \gamma_{\mathrm{L} 0}=0.8$ and $v_{\mathrm{d}} / \nu_{\mathrm{f}}=5$. The value of $v_{\mathrm{d}} / \gamma_{\mathrm{L} 0}$ is 0.50 for steady $(b), 0.30$ for periodic $(c)$ and 0.23 for chaotic $(d)$. Each simulation corresponds to a point in figure 6 . Each spectrogram in this paper has a logarithmic colour code, which spans three orders of magnitude.

We label only periodic, bursty, intermittent and chaotic chirping solutions as hooking or not, since long-lived holes and clumps are trivially hooked.

The quantitative definition and the numerical implementation of these sub-categories of chirping are described in appendix B. The thresholds in our categorization algorithm are adjusted empirically.

\subsection{Typical nonlinear behaviour}

For a typical example of each nonlinear regime, figures 2-5 show the time evolution of the electric field amplitude. For each example, we also show the spectrogram $\mathcal{P}(\delta \omega, t)$ obtained with a shifting Fourier window of size $\Delta t_{\mathcal{F}}=512$ and $N_{\mathcal{F}}=16$ points per window.

Figure 2 shows three nonlinear regimes, which do not feature frequency sweeping, namely steady, periodic and chaotic. These solutions were predicted and observed when collisions are modelled by a Krook operator, for sufficiently large collisions.

Figure 3 shows three nonlinear regimes with frequency sweeping, namely periodic chirping, bursty chirping and intermittent chirping, which are not obtained in the Krook case. The electric field amplitude displays clear relaxation 

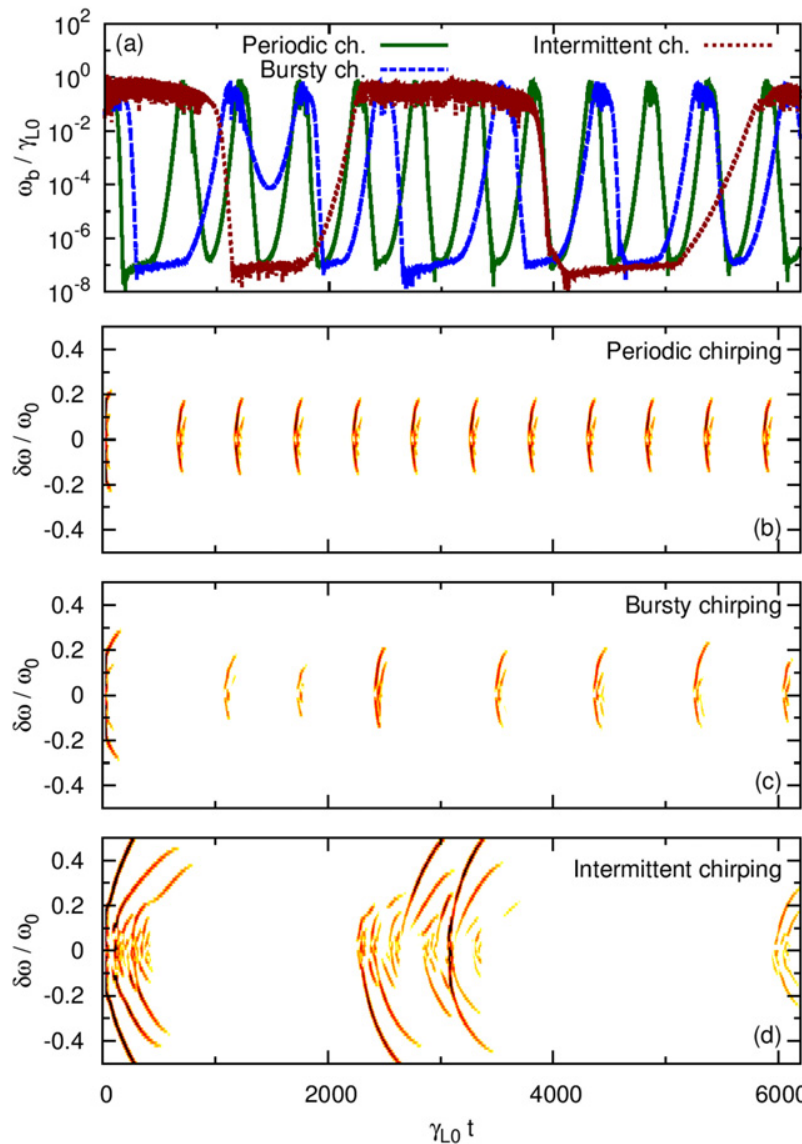

Figure 3. (a) Time series of electric field amplitude and $(b),(c),(d)$ spectrograms for typical chirping solutions with $\gamma_{\mathrm{d}} / \gamma_{\mathrm{L} 0}=0.5$ and $v_{\mathrm{d}} / \nu_{\mathrm{f}}=5$. The value of $v_{\mathrm{d}} / \gamma_{\mathrm{L} 0}$ is 0.14 for periodic chirping $(b)$, 0.11 for bursty chirping (c) and 0.065 for intermittent chirping $(d)$. Each simulation corresponds to a point in figure 6 .

oscillations, with more or less regularity depending on the parameters. The periodic chirping regime of the BB model was discovered and studied in detail in [23].

Figure $4(b)$ shows a fourth nonlinear regime with frequency sweeping, namely chaotic chirping. This is also an example of a solution with upward chirping dominant. In general, chaotic chirping corresponds to an uninterrupted generation of holes and clumps of various sizes, which yields a spectrogram with many minor and major chirping branches without clear quiescent phases. This latter behaviour is the only chirping behaviour that was obtained in the Krook case. There are also other kinds of chaotic chirping, not shown here, where the spectrogram features several behaviours alternating in time, for example bursty chirping and wavering hole.

For $v_{\mathrm{f}} \sim v_{\mathrm{d}}$, dynamical friction significantly modifies the shape of a chirping branch, up to a situation where the sweeping direction of a chirping hole is reversed at some point, as seen in figure $4(b)$, which is called hooked chirping. For larger collision frequencies, the contradicting effects of drag and dissipation on a hole seem to balance, yielding long-lived holes with a more or less stable frequency shift. Such situations have been observed and explained in [22]. Figure 5 shows three nonlinear regimes with long-lived holes. When several holes coexist, the categorization into steady, oscillatory or wavering holes is based on the hole with the largest frequency shift,
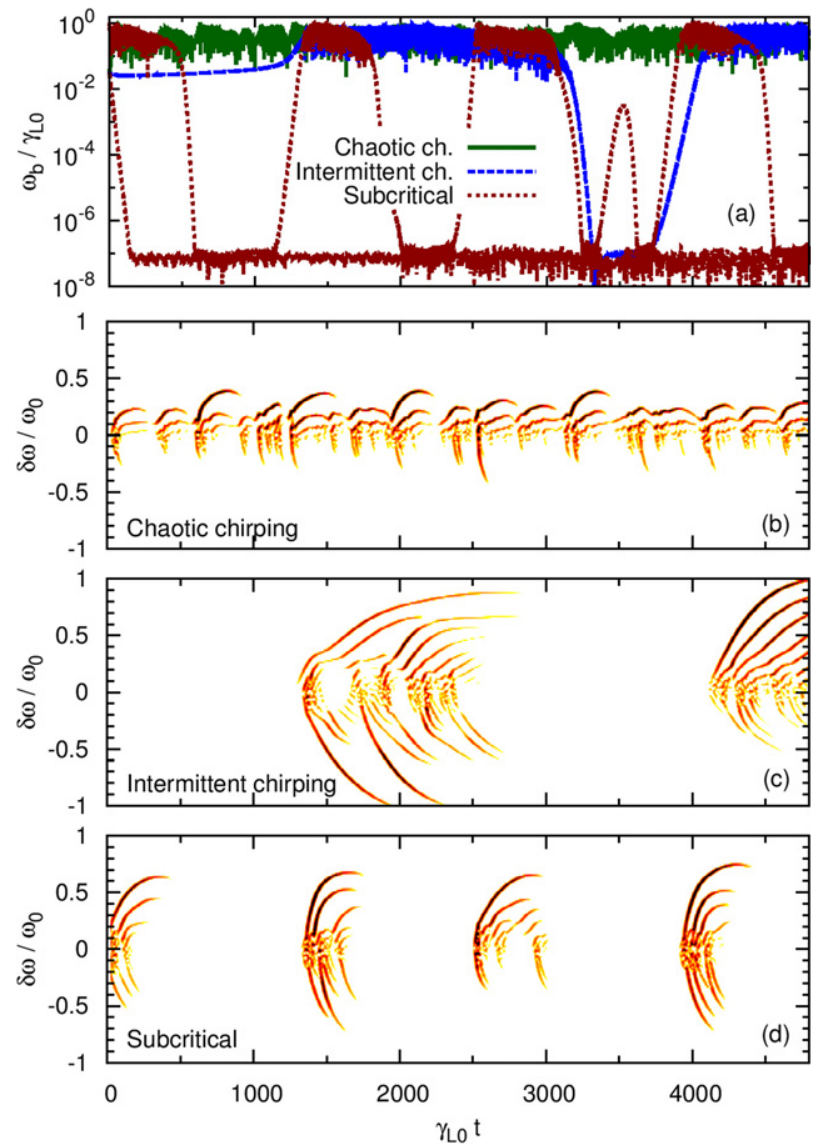

Figure 4. (a) Time series of electric field amplitude and $(b),(c),(d)$ spectrograms for typical chirping solutions with $v_{\mathrm{f}}=v_{\mathrm{d}}$. The parameters are $\gamma_{\mathrm{d}} / \gamma_{\mathrm{L} 0}=0.8, \nu_{\mathrm{d}} / \gamma_{\mathrm{L} 0}=0.13$ for chaotic chirping $(b), \gamma_{\mathrm{d}} / \gamma_{\mathrm{L} 0}=1.0, v_{\mathrm{d}} / \gamma_{\mathrm{L} 0}=0.05$ for intermittent chirping $(c)$, and $\gamma_{\mathrm{d}} / \gamma_{\mathrm{L} 0}=1.2, \nu_{\mathrm{d}} / \gamma_{\mathrm{L} 0}=0.09$ for a subcritical case $(d)$. In the subcritical case, we show the amplitude time-series for two choices of initial amplitude $\omega_{\mathrm{b}}(0) / \gamma_{\mathrm{L} 0}: 0.03$, which yields a damped solution, and 1.0, which yields a nonlinear instability. Each simulation corresponds to a point in figure 7 .

which is observed to dominate the spectrogram. We observed that the number of holes roughly increases with decreasing collisionality.

In addition to these examples of each category, we mention two interesting situations. In figure $4(d), \gamma_{\mathrm{d}} / \gamma_{\mathrm{L} 0}=1.2$ and the growth rate is negative, $\gamma / \gamma_{\mathrm{L} 0}=-0.18$. Linear theory predicts that a wave with a small amplitude is damped exponentially, which is what we observe for a small initial perturbation amplitude $\omega_{\mathrm{b}} / \gamma_{\mathrm{L} 0}=0.03$. However, if we raise the initial perturbation amplitude to $\omega_{\mathrm{b}} / \gamma_{\mathrm{L} 0}=1$, although the solution initially follows linear theory, it then reverses and grows to a chirping state with a saturation level comparable to supercritical solutions. This subcritical instability was first observed for the BB model in [19]. The subcritical solution that we show also happens to be an example of hooked chirping, although close to the boundary between hook and no-hook regimes, with chirping branches only slightly hooking. In figure $4(c)$ and the corresponding amplitude time series in figure 4(a), it appears that the wave is steady for a long time, before growing explosively to a chirping state. In this case, the growth rate is very small, $\gamma / \gamma_{\mathrm{L} 0}=1.5 \times 10^{-7}$. Since the wave amplitude grows faster than the exponential growth 

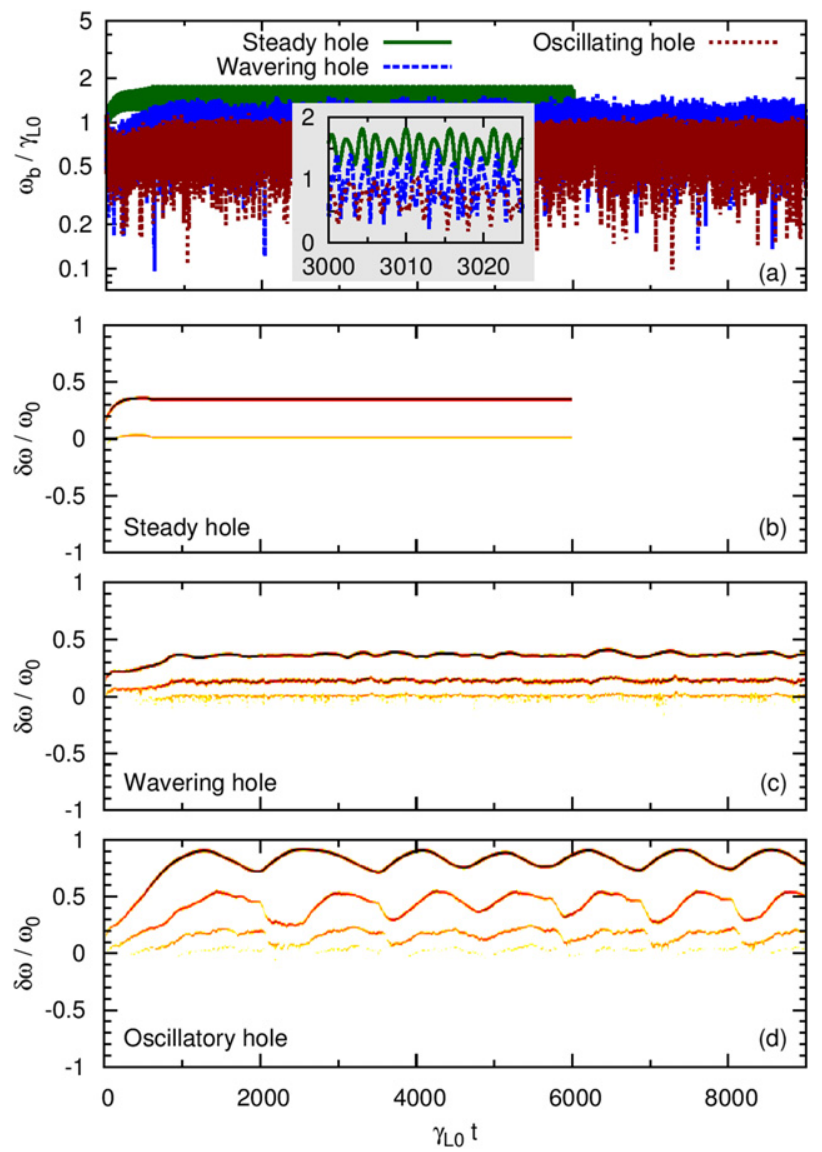

Figure 5. (a) Time series of electric field amplitude and $(b),(c),(d)$ spectrograms for typical solutions with long-lived hole, with $\gamma_{\mathrm{d}} / \gamma_{\mathrm{L} 0}=0.5$. The parameters are $\nu_{\mathrm{f}} / \gamma_{\mathrm{L} 0}=0.36, \nu_{\mathrm{d}} / \gamma_{\mathrm{L} 0}=0.27$ for steady hole $(b), v_{\mathrm{f}} / \gamma_{\mathrm{L} 0}=0.16, v_{\mathrm{d}} / \gamma_{\mathrm{L} 0}=0.09$ for wavering hole $(c)$ and $\nu_{\mathrm{f}} / \gamma_{\mathrm{L} 0}=0.16, \nu_{\mathrm{d}} / \gamma_{\mathrm{L} 0}=0.12$ for oscillatory hole $(d)$. Inset: zoom over a few oscillation periods of the amplitude. Each simulation corresponds to a point in figure 9.

predicted by linear theory, we can conclude that the growth of the wave is nonlinear. This nonlinear instability with $\gamma>0$ is observed for the first time. For $v_{\mathrm{f}}=v_{\mathrm{d}}=0.05 \gamma_{\mathrm{L} 0}$, we observe nonlinear instabilities when $\gamma / \gamma_{\mathrm{L} 0}<0.04$. In the same way as in the subcritical regime, the stability depends on the initial perturbation amplitude. A consequence for experiments is that, in both subcritical and supercritical cases, the wave saturates to a high level $\omega_{\mathrm{b}} \sim \gamma_{\mathrm{L}}$ much sooner than what linear theory predicts $\left(\gamma_{L} t \sim 10^{3}\right.$ instead of $10^{8}$ for this explosive case and $10^{2}$ instead of $\infty$ for the subcritical case). The mechanisms of subcritical and supercritical nonlinear instabilities seem to be the same, which will be explained in a future work. In a long-time point of view though, after nonlinear saturation, the chirping behaviour does not depend on the initial amplitude. The case in figure $4(c)$ is not a new regime, rather an intermittent chirping case. Although nonlinear instabilities require large seed perturbations, they are relevant to experiments, where changes in equilibrium profiles may bring a linearly unstable wave, which is saturated to a relatively large amplitude, to a linearly stable state. If the system alternates between supercritical and subcritical regimes, hysteresis is expected.

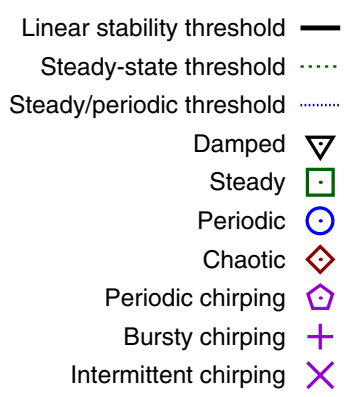

Linear stability threshold -

Steady-state threshold $\cdots . .$.

Steady/periodic threshold .........

Damped $\nabla$

Steady $\square$

Periodic $\odot$

Chaotic $\diamond$

Periodic chirping $\Delta$

Bursty chirping +

Intermittent chirping $X$

Chaotic chirping 米

Steady hole

Oscillatory hole

Wavering hole

Upward chirping dominant $\Delta$

Downward chirping dominant $\nabla$

Upward chirping only $\Delta$

Downward chirping only

Hooked

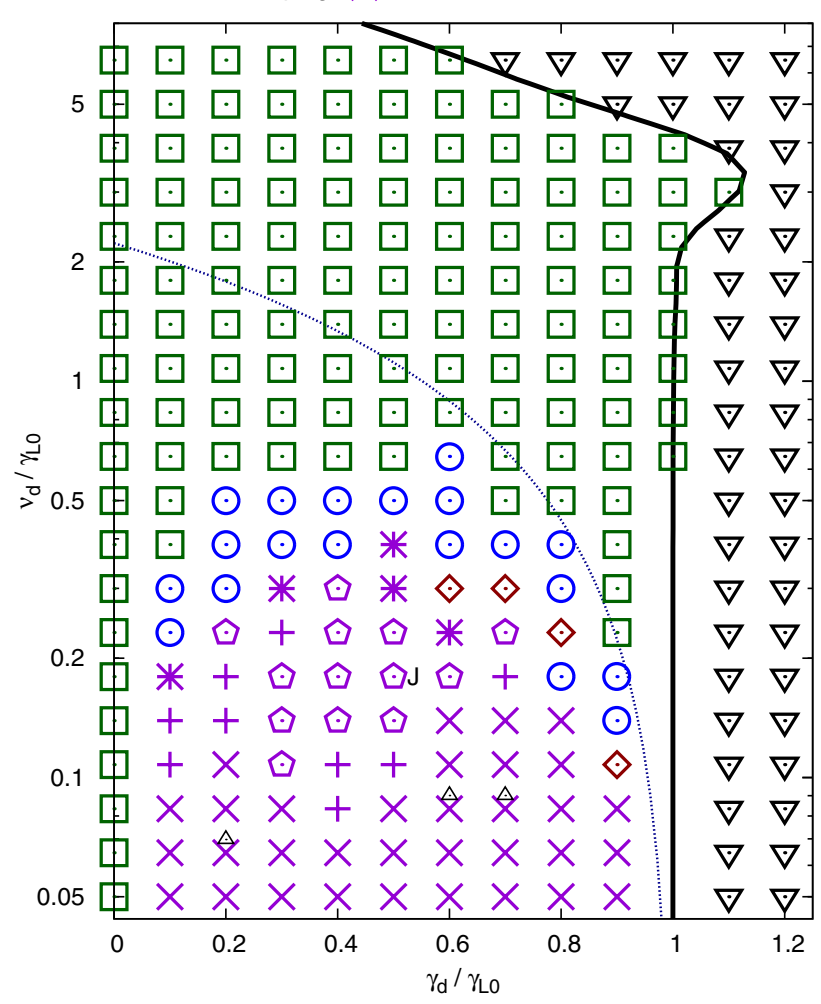

Figure 6. Behaviour bifurcation diagram for $\gamma_{\mathrm{L} 0}=0.1$ and $v_{\mathrm{d}} / v_{\mathrm{f}}=5$. The classification of each solution is plotted in the $\left(\gamma_{\mathrm{d}}, v_{\mathrm{d}}\right)$ parameter space. The legend is shared between figures 6-9. An absence of point signifies that longer, or better resolved simulations are necessary to categorize the time-asymptotic behaviour. The letter J indicates the JT-60U discharge E32359.

\subsection{Small drag}

To investigate the regime of small drag, we perform a series of 260 simulations where $v_{\mathrm{d}} / \nu_{\mathrm{f}}=5$, which is relevant to present-day tokamaks [23]. Figure 6 shows the categorization of each simulation result in the $\left(\gamma_{\mathrm{d}}, \nu_{\mathrm{d}}\right)$ parameter space. The linear growth rate $\gamma$ is obtained by solving the eigenvalue problem corresponding to the linearized model equations. Note the agreement between the linear stability threshold $\gamma=0$ and the boundary between linearly stable and unstable simulations. This phase diagram is qualitatively similar to what was obtained with Krook collisions. We recover the same bifurcations from steady, to periodic, to chaotic, to chirping as the collision frequencies decrease. We do not observe chirping solutions for $\gamma_{\mathrm{d}} / \gamma_{\mathrm{L} 0}<0.1$. The main difference is that chirping solutions can be intermittent, bursty or periodic, whereas we only observed chaotic chirping in the Krook case. We observed that several holes and clumps with different amplitudes coexist in the Krook case, while diffusion smooths 
out fine-scale structures, which explains isolated chirping events we observe in the drag-diffusion case. Nonlinear instabilities, including subcritical instabilities, are also found when the initial amplitude is raised to $\omega_{\mathrm{b}}(t=0) / \gamma_{\mathrm{L} 0}=1$, for collision frequencies slightly below the range of our diagram, $v_{\mathrm{d}} / \gamma_{\mathrm{L} 0}<0.04$. Based on the analysis of [23], toroidal AEs (TAEs) in JT-60U discharge E32359 are roughly located at the point marked by the letter $J$ in this diagram. In such a case, the strategy to avoid chirping bursts would be to increase the ratio $\gamma_{\mathrm{d}} / \gamma_{\mathrm{L}}$, perhaps by decreasing the beam injection power, in order to bring the system closer to marginal stability. Another approach would be to increase the effective collision frequencies, but the required increase may be too difficult to achieve.

When $\gamma \ll \gamma_{\mathrm{L} 0}$, a reduced integral equation for the time evolution of the electric field amplitude was developed using an extension based on the closeness to marginal stability [13], in the case with Krook and diffusion collisions. Lilley included drag into the reduced equation, and found that it does not admit any steady solution when $v_{\mathrm{d}} / v_{\mathrm{f}}<1.043$ [22]. This steady-state threshold is shown in the bifurcation diagrams, when relevant. When a steady-state solution does exist, nonlinear stability analysis reveals that it is unstable when $v_{\mathrm{d}}<v_{\mathrm{cr}}\left(v_{\mathrm{f}}\right)$, where $v_{\mathrm{cr}}\left(v_{\mathrm{f}}\right)$ can be calculated numerically by solving a dispersion relation shown in the reference. As $v_{\mathrm{d}}$ crosses this threshold from above, a steady-state solution bifurcates to a periodic solution. Therefore, we call $v_{\mathrm{cr}}\left(v_{\mathrm{f}}\right)$ the steady/periodic threshold, and include it in the bifurcation diagrams, when relevant. Figure 6 is consistent with this theory, since the whole behaviour diagram is above the steadystate threshold and does admit steady solutions. It also shows qualitative agreement with the steady/periodic threshold for $\gamma \ll \gamma_{\mathrm{L} 0}$ (here $\gamma / \gamma_{\mathrm{L} 0}=0.5$ corresponds to $\gamma_{\mathrm{d}} / \gamma_{\mathrm{L} 0}=0.44$ for small $\left.v_{\mathrm{d}}\right)$.

\subsection{Large drag}

To investigate the effect of large drag, we perform a series of 221 simulations where $v_{\mathrm{d}} / v_{\mathrm{f}}=1$, which may be more relevant to ITER parameters [22]. Figure 7 shows the categorization of each simulation result in the $\left(\gamma_{\mathrm{d}}, v_{\mathrm{d}}\right)$ parameter space. This diagram is qualitatively different from what was obtained with Krook collisions. We do not observe any steady solution, which is consistent with the theoretical steady-state threshold. Neither do we observe periodic or chaotic solutions. Instead, long-lived holes fill the parameter space for large collision frequencies. For $v_{\mathrm{f}} / \gamma_{\mathrm{L} 0}=v_{\mathrm{d}} / \gamma_{\mathrm{L} 0}<0.1$ and $\gamma_{\mathrm{d}}>\gamma_{\mathrm{L} 0}$, we observe subcritical instabilities, which are stable for $\omega_{\mathrm{b}}(t=0) / \gamma_{\mathrm{L} 0}=0.03$ but unstable for $\omega_{\mathrm{b}}(t=0) / \gamma_{\mathrm{L} 0}=1$. Other stable solutions may be unstable for even larger initial amplitudes. We find only 1 periodic chirping solution, compared with 13 in the small drag case, which suggests that quasi-periodically bursting AEs, which are often observed in current tokamaks, may not or rarely be observed in ITER.

Since the initial distribution is symmetric around the resonant velocity, the drag term is the only term in the $\delta f-\mathrm{BB}$ model that is asymmetric around $\delta \omega=v-1=0$. This is consistent with the fact that we find only three upward chirping dominant solutions when $v_{\mathrm{f}}=v_{\mathrm{d}} / 5$, whereas most solutions are upward chirping dominant or upward chirping only when

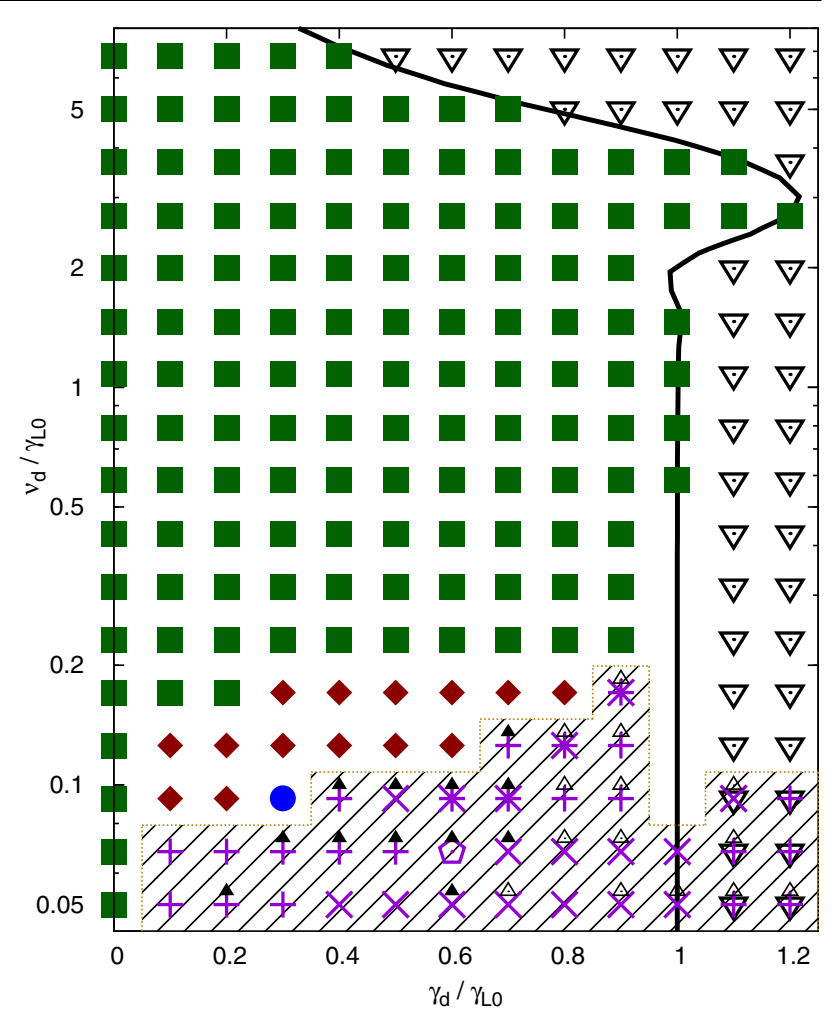

Figure 7. Behaviour bifurcation diagram for $\gamma_{\mathrm{L} 0}=0.1$ and $v_{\mathrm{d}} / v_{\mathrm{f}}=1$. The classification of each solution is plotted in the $\left(\gamma_{\mathrm{d}}, v_{\mathrm{d}}\right)$ parameter space. In the bottom right corner, superposed symbols show subcritical solutions for which the amplitude is damped when $\omega_{\mathrm{b}} / \gamma_{\mathrm{L} 0}=0.03$, but unstable when $\omega_{\mathrm{b}} / \gamma_{\mathrm{L} 0}=1$.

$v_{\mathrm{f}}=v_{\mathrm{d}}$ (steady-hole solutions are trivially asymmetric). Drag has a counter-intuitive effect on chirping asymmetry. Since the effect of drag on any phase-space structure is to advect it from large to small velocities, one could imagine that the presence of drag would make down chirping dominant. However, upward chirping dominates and a physical explanation is proposed in [22].

\subsection{Close to marginal stability}

Let us investigate the barely unstable regime, which is relevant for theory and may be relevant for ITER in some cases [27]. We perform a series of 204 simulations where $\gamma_{\mathrm{d}} / \gamma_{\mathrm{L} 0}=0.9$, which is near marginal stability in the sense that $\gamma / \gamma_{\mathrm{L}} \approx 0.05$. Figure 8 shows the categorization of each simulation result in the $\left(v_{\mathrm{f}}, v_{\mathrm{d}}\right)$ parameter space. The linear stability threshold is out of the range of this plot. As predicted by theory, steadystate solutions exist only above the steady-state threshold. Moreover, the boundary between steady and periodic solutions agrees with the theoretical steady/periodic threshold.

\subsection{Farther from marginal stability}

To investigate the nonlinear behaviour relatively far from marginal stability (from an instantaneous point of view, not necessarily from a time-averaged point of view), we perform a series of 204 simulations where $\gamma_{\mathrm{d}} / \gamma_{\mathrm{L} 0}=0.5$, which is relevant for experiments [23]. Figure 9 shows the categorization of each simulation result in the $\left(v_{\mathrm{f}}, v_{\mathrm{d}}\right)$ 


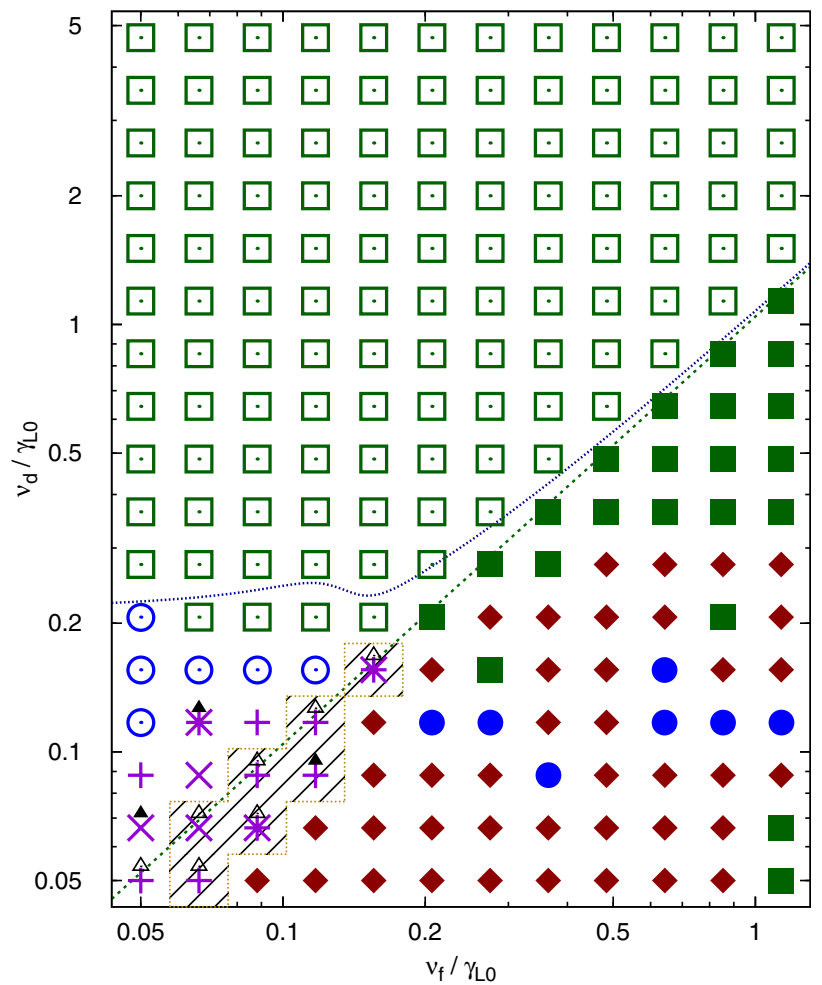

Figure 8. Behaviour bifurcation diagram for $\gamma_{\mathrm{L} 0}=0.1$ and $\gamma_{\mathrm{d}} / \gamma_{\mathrm{L} 0}=0.9$. The classification of each solution is plotted in the $\left(v_{\mathrm{f}}, v_{\mathrm{d}}\right)$ parameter space.

parameter space. The linear stability threshold is out of the range of this plot. The agreement with the theoretical steadystate threshold and steady/periodic threshold is not as good as when $\gamma_{\mathrm{d}} / \gamma_{\mathrm{L} 0}=0.9$, which is expected since for $\gamma_{\mathrm{d}} / \gamma_{\mathrm{L} 0}=0.5$, $\gamma / \gamma_{\mathrm{L} 0} \approx 0.4$.

\subsection{General remarks}

In all cases, each category is relatively isolated in a welldefined region, rather than dispersed in the whole parameter space. Note also that the region of hooked chirping is contiguous to the region of a long-lived hole. These remarks bring more confidence in our categorization algorithm.

All periodic chirping solutions satisfy our criteria for bursty chirping. In this sense, periodic chirping is a special case of bursty chirping. Periodic chirping solutions are restricted to a region where $\gamma_{\mathrm{d}} / \gamma_{\mathrm{L}}=0.2-0.7$, which is relatively far from marginal stability. This suggests that, in AE experiments that feature periodic chirping bursts, one must be careful in using the assumption $\gamma_{\mathrm{d}} \approx \gamma_{\mathrm{L}}$, which stems naively from selforganization arguments.

Although the terms in $v_{\mathrm{f}}$ and $v_{\mathrm{d}}$ in the BB equations are referred to as drag and diffusion, they do not correspond to drag and diffusion in the sense of a 3D Fokker-Planck collision operator, but rather to a projection of these operators on a resonant surface of one $\mathrm{AE}$ for instance. In fact, $v_{\mathrm{f}}$ includes contributions from the slowing-down term, the pitch-angle scattering term and the parallel velocity diffusion, while $v_{\mathrm{d}}$ includes contributions from the pitch-angle scattering term and the parallel velocity diffusion $[22,23]$.

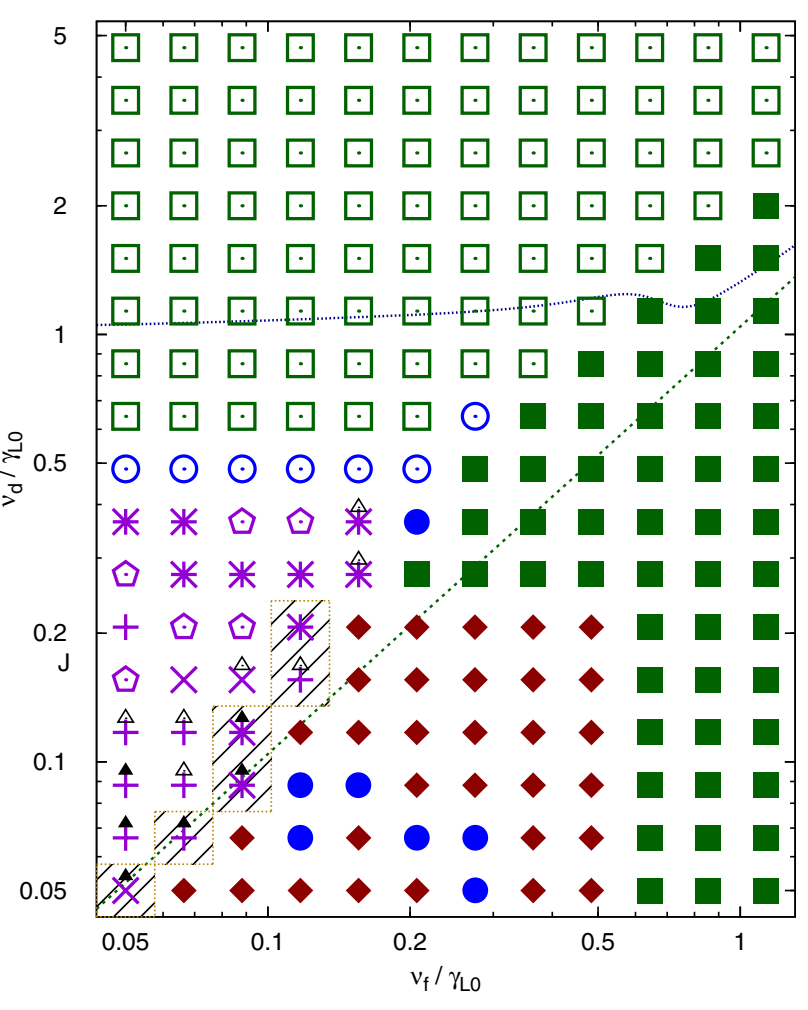

Figure 9. Behaviour bifurcation diagram for $\gamma_{\mathrm{L} 0}=0.1$ and $\gamma_{\mathrm{d}} / \gamma_{\mathrm{L} 0}=0.5$. The classification of each solution is plotted in the $\left(v_{\mathrm{f}}, v_{\mathrm{d}}\right)$ parameter space.

We do not observe either long-lived clump or downchirping dominant cases, which are often observed in the experiment. Although the reason eludes us, we can speculate that down-chirping dominant cases in TAE experiments may be the result of reversed magnetic shear, which effectively brings a minus sign in front of $v_{\mathrm{f}}^{2}$ in equation (1) when the 3D FokkerPlanck operator is projected on the resonant surface. However, there are other possible causes, such as particular shapes of $f_{0}$ with a non-constant slope, departures from the adiabatic bulk hypothesis and processes that are not included in the BB model.

\section{Conclusions}

In this paper, we proposed a qualitative definition for each chirping regime of the BB model with drag and diffusion, and gave the corresponding quantitative criteria. We have developed an algorithm with empirically adjusted thresholds, which systematically categorizes a simulation result, and could also be used to analyse experimental data. We investigated the parameter space for a constant-slope energetic-particle distribution and a wide range of parameters, which includes ITER-relevant collision frequencies, or parameters that can be used to reproduce magnetic signals of JT-60U and MAST experiments.

The above behaviour bifurcation diagrams show, for the first time, the parameter range for each regime, and the relationship between regimes. They indicate how input parameters in $\mathrm{AE}$ experiments and simulations may be modified to reach a regime of interest.

Ultimately, nonlinear behaviour bifurcations may be used to mitigate energetic-particle transport in $\mathrm{AE}$ experiments. 
For example, chirping bursts correspond to self-coherent phase-space structures evolving in the velocity direction in the $\mathrm{BB}$ model, which corresponds to the toroidal angular momentum in the $3 \mathrm{D}$ AE problem. Thus, in experiments, we can speculate that chirping bursts correspond to radially evolving self-coherent structures and be associated with convective transport. However, we must note the following caveats. (1) Although quantitative similarities between AE experiments and the $\mathrm{BB}$ model have been found, a one-toone correspondence has not been established, leaving some uncertainty in any experimental analysis based on the BB model. (2) Processes that are not included in the BB model, such as turbulence-induced drag and diffusion, may change the whole qualitative picture, or yield new kinds of behaviour, such as long-lived clumps if some process brings an effect opposite to collisional drag. (3) Several resonances or several modes coexist in most experiments, and can interact to produce other kinds of behaviour, such as avalanches. The interaction between several resonances is a hot topic currently under investigation.

In addition, we discovered the existence of a nonlinear instability in the barely unstable regime.

\section{Acknowledgments}

The main author thanks X. Garbet, S. Sharapov, B.N. Breizman, M.K. Lilley, and P.H. Diamond for fruitful discussions. This work was supported by the World Class Institute (WCI) Program of the National Research Foundation of Korea (NRF) funded by the Ministry of Education, Science and Technology of Korea (MEST) [WCI 2009-001], by a grant-in-aid for scientific research of JSPF, Japan (21224014), and by the collaboration program of the RIAM of Kyushu University and Asada Science foundation. Computations were performed on the Kraken system at NFRI.

\section{Appendix A. Verification of COBBLES with drag and diffusion}

In [21], COBBLES was verified solely when collisions are modelled by a Krook operator. It is necessary to verify our implementation of drag and diffusion. Firstly, we confirm that, in the absence of an electric field, a Gaussian perturbation in the velocity distribution follows the analytic solution of the Fokker-Planck equation. As an additional test, we compare saturation amplitudes and steady-state solutions between $\delta f$-COBBLES and analytic predictions derived in [28]. Figures A1 and A2 show the saturated electric field amplitude and the perturbed velocity distribution $\delta f(v)=$ $\langle f\rangle-f_{0}$ at $\gamma_{\mathrm{L} 0} t=1000$ in $\delta f$-COBBLES simulations with $\gamma_{\mathrm{L} 0}=0.1, \gamma_{\mathrm{d}} / \gamma_{\mathrm{L} 0}=0.9$ and different collision frequencies. We observe qualitative agreement with theory. The source of the quantitative discrepancy we observe is unknown as yet. It may be explained by a deviation from the condition $\gamma / \gamma_{\mathrm{L} 0} \ll 1$, which is used in theory for the perturbative treatment of the nonlinearities. We ruled out border effects by checking that doubling the velocity range, where the slope of $f_{0}$ is constant $\left(v_{1}=-1, v_{2}=3\right.$ and $\left.N_{v}=4096\right)$, does not significantly reduce the disagreement.

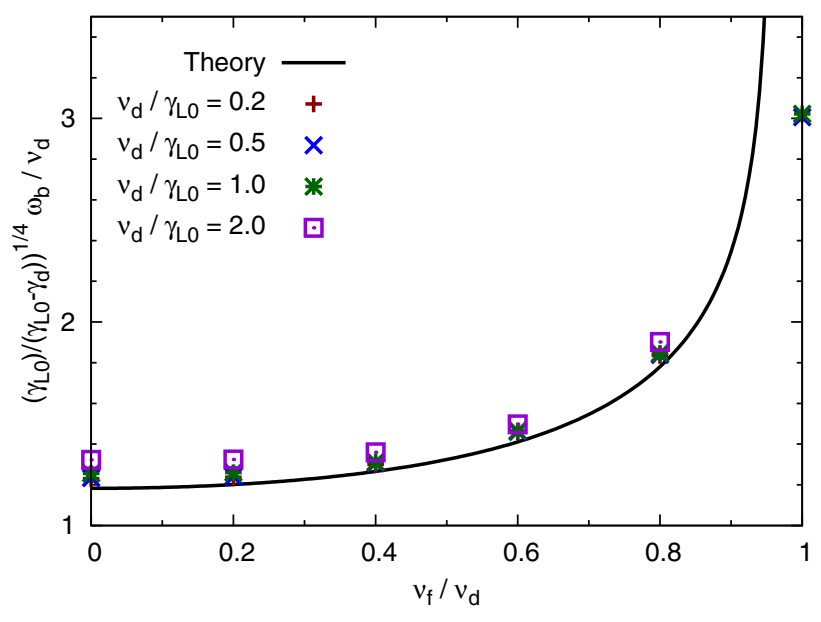

Figure A1. Steady-state saturation level with drag and diffusion. The points correspond to $\delta f$ simulations with $\gamma_{\mathrm{L} 0}=0.1$ and $\gamma_{\mathrm{d}} / \gamma_{\mathrm{L} 0}=0.9$. The solid curve corresponds to analytic theory.

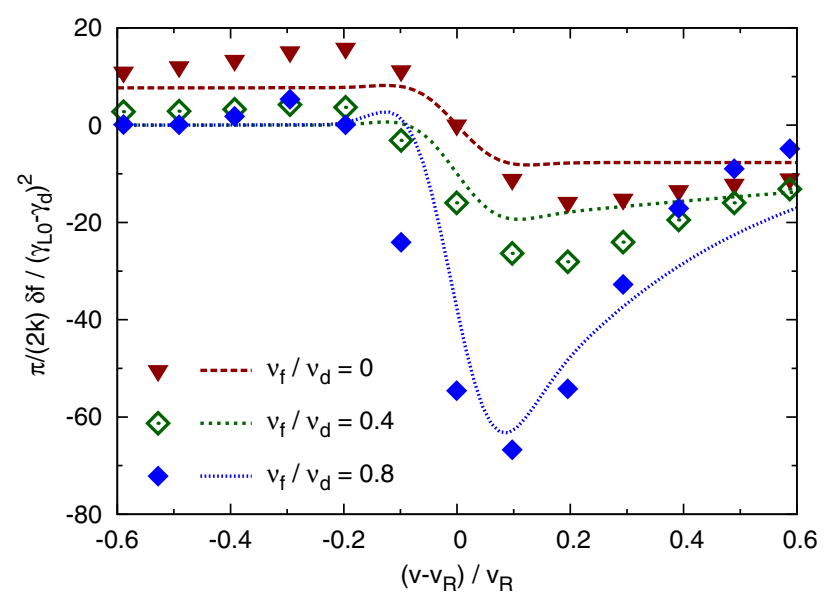

Figure A2. Perturbed velocity distribution in a steady state. The points correspond to $\delta f$ simulations with $\gamma_{\mathrm{L} 0}=0.1, \gamma_{\mathrm{d}} / \gamma_{\mathrm{L} 0}=0.9$ and $v_{\mathrm{d}} / \gamma_{\mathrm{L} 0}=5$. The dashed lines correspond to analytic theory.

\section{Appendix B. Categorization algorithm}

Since we want to categorize the time-asymptotic behaviour, rather than the initial, transient behaviour, we define a time $t_{\min }$ before which the solution is ignored:

$$
t_{\min } \equiv \max \left(t_{\max }, v_{\mathrm{eff}}^{-1}, 5 \frac{2 \pi}{|\gamma|}\right),
$$

where $t_{\max }$ is the total simulation time and $v_{\text {eff }}=$ $\max \left(v_{\mathrm{f}}^{2} / \gamma_{\mathrm{L} 0}, v_{\mathrm{d}}^{3} / \gamma_{\mathrm{L} 0}^{2}\right)$. Hereafter, $\mathcal{P}$ refers to a spectrogram of the electric field at $x=0$, obtained with a shifting Fourier window of size $\Delta t_{\mathcal{F}}=512$ and $N_{\mathcal{F}}=16$ points per window. We write $\mathcal{P}_{j}^{n} \equiv \mathcal{P}\left(\delta \omega_{j}, t_{n}\right)$ the power in this spectrogram at a discrete time $t_{n}=t_{\min }+n \Delta t$ and for a discrete frequency shift $\delta \omega_{j}=j \Delta \omega$, where $\Delta t=\Delta t_{\mathcal{F}} / N_{\mathcal{F}}$, and $\Delta \omega=2 \pi / \Delta t_{\mathcal{F}}$. $\mathcal{P}_{\max }^{\text {glob }}$ is the global maximum of $\mathcal{P}$ for $t>t_{\min }$.

To analyse $\mathcal{P}$, we track chirping branches in the following way. To simplify the explanations, let us consider the $\delta \omega \geqslant 0$ part of $\mathcal{P}$ only. For each time step $n \geqslant 0$, we search for local maxima $\mathcal{P}_{j}^{n}>\mathcal{P}_{j \pm 1}^{n}$ and interpolate the corresponding values 
of $\mathcal{P}$ and $\delta \omega$ :

$$
\mathcal{P}_{\max }^{n}=\mathcal{P}_{j}^{n}-\frac{\left(\mathcal{P}_{j+1}^{n}-\mathcal{P}_{j-1}^{n}\right)^{2} / 8}{\mathcal{P}_{j-1}^{n}-2 \mathcal{P}_{j}^{n}+\mathcal{P}_{j+1}^{n}}
$$

and

$$
\delta \omega_{\max }^{n}=\delta \omega_{j}-\frac{\Delta \omega}{2} \frac{\mathcal{P}_{j+1}^{n}-\mathcal{P}_{j-1}^{n}}{\mathcal{P}_{j-1}^{n}-2 \mathcal{P}_{j}^{n}+\mathcal{P}_{j+1}^{n}} .
$$

We keep only those maxima for which $\mathcal{P}_{\max }^{n}>\alpha_{\mathrm{p}} \mathcal{P}_{\max }^{\text {glob }}$, with $\alpha_{\mathrm{p}}=0.05$. To track a chirping branch $\delta \omega^{n}$, we search for maxima that are contiguous in time. For each $n \geqslant 0, \delta \omega^{n+1}$ takes the value of the local maximum $\delta \omega_{\max }^{n+1}$ the closest to $\delta \omega^{n}$. Initially, we lock on the maximum with the largest $\delta \omega$ (by initializing $\delta \omega^{-1}$ to a very large positive value). If one of the two following conditions is met, the current chirping branch is considered to be terminated and the tracking of the next chirping branch starts: (1) there is no local maximum as defined above; or (2) the difference is much larger than a theoretical prediction [17]:

$$
\Delta \omega_{\mathrm{cut}}(\delta \omega)=\frac{256}{27 \pi^{4}} \frac{\gamma_{\mathrm{L} 0}^{2} \gamma_{\mathrm{d}}}{\delta \omega} \Delta t
$$

more precisely $\left|\delta \omega^{n+1}-\delta \omega^{n}\right| / \alpha_{\text {cut }}>\Delta \omega_{\text {cut }}\left(\delta \omega^{n}\right)$, $\Delta \omega_{\text {cut }}\left(\delta \omega^{n+1}\right)$, with $\alpha_{\text {cut }}=5$.

Independently, we define $\delta \omega_{\max }^{\text {glob }}$ as the maximum of $\delta \omega$ for which $\mathcal{P}$ is a local maximum that satisfies $\mathcal{P}>\epsilon_{\mathrm{p}} \mathcal{P}_{\text {max }}^{\text {glob }}$, with $\epsilon_{\mathrm{p}}=0.01$.

\section{B.1. Recurrence effect and chirping}

To categorize a solution as damped, steady state, periodic, chaotic or chirping, we use a slightly improved version of an algorithm, which was described and benchmarked in [21]. There are two improvements.

Firstly, as can be seen in figure $3(a)$, periodic, bursty and intermittent chirping (which do not appear in former works with a Krook collision operator) could be mistaken for solutions that suffer from a spurious recurrence effect. To distinguish these two situations, we compare the theoretical recurrence time $T_{\mathrm{R}}=2 \pi / \Delta v$, where $\Delta v$ is the velocity grid size, with the times where the amplitude grows back from a numerical noise level to a significant level. More precisely, if $\omega_{\mathrm{b}}(t)$ decays below a threshold $\epsilon_{0}=10^{-7}$, the solution is damped, unless there is a time $t_{\mathrm{r}}$ such as $0.45 T_{\mathrm{R}}<t_{\mathrm{r}}\left(\bmod T_{\mathrm{R}}\right)<0.55 T_{\mathrm{R}}$, for which $\omega_{\mathrm{b}}\left(t_{\mathrm{r}}\right)>\alpha_{0}$, with $\alpha_{0}=10^{-4}$. A caveat is that periodic chirping with a period close to the recurrence time is spuriously identified as damped, but we checked that this did not happen in our analysis.

Secondly, as can be seen in figure 2(c), some non-chirping solutions can have a spectrogram with significant power for $|\delta \omega| / \omega_{0} \approx 0.01-0.05$, which is why we previously defined the threshold for chirping at 0.05 . On the other hand, some chirping solutions have an amplitude of frequency shift as small as $|\delta \omega| / \omega_{0} \approx 0.04$, which were spuriously categorized as not chirping. To remedy this issue (using our chirping branch tracking method), a solution is now identified as chirping if and only if there is a chirping branch such that $\left|\delta \omega_{n}\right| / \omega_{0}>\epsilon_{7}$ for some $n \geqslant 0$. We choose $\epsilon_{7}=0.03$.

\section{B.2. Kind of chirping}

To categorize chirping solutions, we analyse the chirping branches as follows. We count the number of chirping bursts $N_{\text {bursts }}$, defined as the number of time where a chirping branch crosses a threshold $\delta \omega^{n}=\alpha_{\text {burst }} \delta \omega_{\text {max }}^{\text {glob }}$ from below, where $\alpha_{\text {burst }}=0.4$. We retrieve the maximum duration of a chirping branch $T_{\max }^{\text {chirping }}$, the cumulated duration of all chirping branches $T_{\text {total }}^{\text {chiring }}$, the maximum duration of a quiescent phase $T_{\max }^{\text {quiescent }}$ and the cumulated duration of all quiescent phases $T_{\text {total }}^{\text {quiescent }}$, where a quiescent phase is defined as a time interval between two successive chirping branches. To sort out whether a long-lived hole is steady, oscillatory or chaotic, we take the longest chirping branch $\delta \omega_{\text {longest }}^{n}$, and use basically the same algorithm as the one we use to categorize the time series of the electric field amplitude, but for $\delta \omega_{\text {longest }}^{n}$ instead of $\omega_{\mathrm{b}}\left(t_{n}\right)$. In other words we define $W\left(t_{n}\right) \equiv \delta \omega_{\text {longest }}^{n}$, its mean value $\langle W\rangle$, maximum $W_{\max }$ and minimum $W_{\min }$, the oscillation amplitude $\Delta W \equiv W_{\max }-W_{\min }$, and the local minima (maxima) as points where $W\left(t_{n}\right)$ is smaller (larger) than $W\left(t_{n-1}\right)$ and $W\left(t_{n+1}\right)$. As a measure of the periodicity, we compute the two-point correlation function as

$$
R(\tau)=\frac{1}{m} \sum_{l=0}^{m-1} \frac{\left\langle\tilde{W}_{l}\left(\tau, \tau^{\prime}\right) \tilde{W}_{l+1}\left(\tau, \tau^{\prime}\right)\right\rangle}{\sqrt{\left\langle\tilde{W}_{l}\left(\tau, \tau^{\prime}\right)^{2}\right\rangle} \sqrt{\left\langle\tilde{W}_{l+1}\left(\tau, \tau^{\prime}\right)^{2}\right\rangle}},
$$

where, for each correlation window size $\tau$,

$\tilde{W}_{l}\left(\tau, \tau^{\prime}\right) \equiv W\left(t_{\text {branch }}-l \tau-\tau^{\prime}\right)-\left\langle W\left(t_{\text {branch }}-l \tau\right)\right\rangle$,

$m=t_{\text {branch }} / \tau$ is the number of period included in the duration $t_{\text {branch }}$ of the chirping branch, and the angular brackets represent the time average over a period,

$$
\left\langle\tilde{W}\left(\tau, \tau^{\prime}\right)\right\rangle=\frac{1}{\tau} \int_{0}^{\tau} \tilde{W}\left(\tau, \tau^{\prime}\right) \mathrm{d} \tau^{\prime} .
$$

The overall correlation $R_{0}$ is defined as the maximum of $R(\tau)$ for $\tau>\tau_{c}$, where $\tau_{c}$ is the shortest period such that $R\left(\tau_{c}\right) \leqslant 0$. In other words, $R_{0}$ is the normalized amplitude of the peak in the two-point correlation function corresponding to the dominant frequency.

We then proceed to the following decision tree:

4.A. IF $T_{\text {total }}^{\text {quiescent }} / T_{\text {total }}^{\text {chirping }}<\epsilon_{11}$ AND $N_{\text {bursts }}<\epsilon_{12} T_{\text {total }}^{\text {chirping }} /$ $T_{\max }^{\text {chirping }}$ THEN long-lived hole

(a) IF each minima (maxima) is larger (smaller) than the former OR $\Delta W /\langle W\rangle<\epsilon_{13}$ THEN steady hole

(b) ELSE IF $R_{0}>1-\epsilon_{14}$ THEN oscillatory hole

(c) ELSE IF the number of extrema is not less than four THEN wavering hole

(d) ELSE failure of the categorization

4.B. ELSE IF $R_{0}^{\text {whole }}>1-\epsilon_{15}$ THEN periodic chirping

4.C. ELSE IF $\left|T_{\text {total }}^{\text {chiring }} /\left(N_{\text {bursts }} T_{\max }^{\text {chirping }}\right)-1\right|<\epsilon_{16}$ THEN bursty chirping

4.D. ELSE IF $T_{\text {total }}^{\text {quiescent }}>\epsilon_{17} T_{\text {total }}^{\text {chiring }}$ THEN intermittent chirping

4.E. ELSE chaotic chirping,

where $R_{0}^{\text {whole }}$ is defined in the same way as $R_{0}$, but for the whole time series that comprises all chirping branches instead of the longest chirping branch only. The thresholds are empirically adjusted to $\epsilon_{11}=0.2, \epsilon_{12}=0.5, \epsilon_{13}=0.01, \epsilon_{14}=0.25$, $\epsilon_{15}=0.25, \epsilon_{16}=0.3$ and $\epsilon_{17}=0.5$. 


\section{B.3. Chirping asymmetry}

We define the total upward power $\mathcal{P}_{\text {total }}^{\text {up }} \equiv \sum_{n, j>0} \mathcal{P}_{j}^{n}$, the total downward power $\mathcal{P}_{\text {total }}^{\text {down }} \equiv \sum_{n, j<0} \mathcal{P}_{j}^{n}$, the maximum frequency shift reached by any upward chirping branch, $\delta \omega_{\max }^{\text {up }}$ and the maximum frequency shift reached by any downward chirping branch, $\delta \omega_{\max }^{\mathrm{down}}$. We then proceed to the following decision tree:

- IF $\mathcal{P}_{\text {total }}^{\text {up }}>\alpha_{\mathrm{a}} \mathcal{P}_{\text {total }}^{\text {down }}$ AND $\left|\delta \omega_{\text {max }}^{\text {down }}\right| / \omega_{0}>\epsilon_{7}$ THEN upward chirping dominant

- ELSEIF $\mathcal{P}_{\text {total }}^{\text {up }}>\alpha_{\mathrm{a}} \mathcal{P}_{\text {total }}^{\text {down }}$ THEN upward chirping only

- ELSEIF $\mathcal{P}_{\text {total }}^{\text {down }}>\alpha_{\mathrm{a}} \mathcal{P}_{\text {total }}^{\text {up }}$ AND $\delta \omega_{\max }^{\text {up }} / \omega_{0}>\epsilon_{7}$ THEN downward chirping dominant

- ELSEIF $\mathcal{P}_{\text {total }}^{\text {down }}>\alpha_{\mathrm{a}} \mathcal{P}_{\text {total }}^{\text {up }}$ THEN downward chirping only

- ELSE symmetric,

with $\alpha_{\mathrm{a}}=3$.

\section{B.4. Hooks}

Hooks are identified by the following decision tree:

- IF there is a chirping branch of maximum frequency shift $\delta \omega_{\text {branch }}$ and an $n$ for which $\delta \omega^{n}<\left(1-\epsilon_{\mathrm{h}}\right) \delta \omega_{\text {branch }}$ and $\delta \omega^{n}<\delta \omega_{\text {branch }}-\Delta \omega$ and $\delta \omega^{n}>n_{\mathrm{h}} \Delta \omega$ and $\delta \omega^{n}>$ $\alpha_{\mathrm{h}} \delta \omega_{\text {branch }}$ THEN hooked

- ELSE no hook,

with $\epsilon_{\mathrm{h}}=0.01, \alpha_{\mathrm{h}}=0.5$ and $n_{\mathrm{h}}=10$.

\section{References}

[1] Fasoli A. et al 1997 Plasma Phys. Control. Fusion 39 B287

[2] Kusama Y. et al 1999 Nucl. Fusion 39 1837-43

[3] Podestà M., Bell R.E., Fredrickson E.D., Gorelenkov N.N., LeBlanc B.P., Heidbrink W.W., Crocker N.A., Kubota S. and Yuh H. 2010 Phys. Plasmas 17122501

[4] Chen W. et al 2010 Nucl. Fusion 50084008

[5] Berk H. et al 2006 Nucl. Fusion 46 S888

[6] Nazikian R. et al 2008 Phys. Rev. Lett. 101185001
[7] Toi K., Ogawa K., Isobe M., Osakabe M., Spong D.A. and Todo Y. 2011 Plasma Phys. Control. Fusion 53024008

[8] Gryaznevich M.P. and Sharapov S.E. 2004 Plasma Phys. Control. Fusion 46 S15

[9] Berk H.L., Breizman B.N. and Pekker M.S. 1997 Plasma Phys. Rep. 23 778-88

[10] Breizman B.N., Berk H.L., Pekker M.S., Porcelli F., Stupakov G.V. and Wong K.L. 1997 Phys. Plasmas 4 1559-68

[11] Wong H.V. and Berk H.L. 1998 Phys. Plasmas 5 2781-96

[12] Berk H.L., Breizman B.N. and Ye H. 1993 Phys. Fluids B 5 1506-15

[13] Berk H.L., Breizman B.N. and Pekker M. 1996 Phys. Rev. Lett. 76 1256-9

[14] Pinches S.D., Berk H.L., Gryaznevich M.P., Sharapov S.E. and the JET-EFDA Contributors 2004 Plasma Phys. Control. Fusion 46 S47-57

[15] Fasoli A., Breizman B.N., Borba D., Heeter R.F., Pekker M.S. and Sharapov S.E. 1998 Phys. Rev. Lett. 81 5564-7

[16] Heeter R.F., Fasoli A.F. and Sharapov S.E. 2000 Phys. Rev. Lett. 85 3177-80

[17] Berk H.L., Breizman B.N. and Petviashvili N.V. 1997 Phys. Lett. A $234213-8$

[18] Berk H.L., Breizman B.N. and Petviashvili N.V. 1998 Phys. Lett. A 238408

[19] Berk H.L., Breizman B.N., Candy J., Pekker M. and Petviashvili N.V. 1999 Phys. Plasmas 6 3102-13

[20] Vann R.G.L., Dendy R.O., Rowlands G., Arber T.D. and d'Ambrumenil N. 2003 Phys. Plasmas 10 623-30

[21] Lesur M., Idomura Y. and Garbet X. 2009 Phys. Plasmas 16092305

[22] Lilley M.K., Breizman B.N. and Sharapov S.E. 2009 Phys. Rev. Lett. 102195003

[23] Lesur M., Idomura Y., Shinohara K., Garbet X. and the JT-60 Team 2010 Phys. Plasmas 17122311

[24] Gorelenkov N., Bernabei S., Cheng C., Hill K., Nazikian R., Kaye S., Kusama Y., Kramer G., Shinohara K., Ozeki T. and Gorelenkova M. 2000 Nucl. Fusion 401311

[25] Heidbrink W.W., Fredrickson E., Gorelenkov N.N., Hyatt A.W., Kramer G. and Luo Y. 2003 Plasma Phys. Control. Fusion 45983

[26] Berk H.L., Breizman B.N. and Pekker M. 1995 Phys. Plasmas 2 3007-16

[27] Gorelenkov N., Berk H. and Budny R. 2005 Nucl. Fusion 45226

[28] Lilley M.K. 2009 Resonant interaction of fast particles with Alfvén waves in spherical tokamaks $P h D$ Thesis Imperial College, UK 